\title{
SOME FUNCTIONAL EQUATIONS IN THE THEORY OF DYNAMIC PROGRAMMING. I. FUNCTIONS OF POINTS AND POINT TRANSFORMATIONS
}

\author{
BY \\ R. BELLMAN
}

1. Introduction. The purpose of this paper is to establish a number of basic results concerning the solutions of a large and important class of functional equations which arise in the theory of dynamic programming. Here we shall be concerned only with the analytic aspects of these equations, and refer the reader to a number of sources, $[2 ; 3 ; 6 ; 7 ; 9 ; 11]$, where the underlying processes are discussed in connection with various applications.

We shall in this paper consider only functions of points and point transformations. In a subsequent one we shall treat more general equations involving functionals and operators.

Let $p$ denote a vector representing a point in an $N$-dimensional region $D$, and $T(p, q)$, for all vectors $q$ in some prescribed $M$-dimensional set $S$, which may consist of a finite number of points, an enumerably infinite set, or which may be a continuum, denote a transformed point lying in $D$ for each $p$ in $D$. Let $f(p)$ denote a scalar function of $p$ and $g(p, q)$ and $h(p, q)$ be two scalar functions of $p$ and $q$.

A representative example of functional equations we shall discuss below is

$$
f(p)=\operatorname{Sup}_{q}[g(p, q)+h(p, q) f(T(p, q))] .
$$

This is an analytic equivalent of the "principle of optimality" for multi-stage decision processes; $c f .[2 ; 11]$.

Apart from their interest in connection with multi-stage decision processes, these equations possess the merit of constituting in many ways a natural extension of linear equations. As such they serve as a valuable bridge between the well-regulated preserve of linear equations and the as yet untamed jungle of nonlinear equations.

In our discussion of these equations, we shall begin with a simple and easily derived inequality relating to the operator in (1) above. This inequality plays a paramount rôle in all our proofs of existence and uniqueness. In the following four sections we shall consider two important subclasses of the equation in (2). Although the existence and uniqueness proofs for each of these classes follow a similar track, each requires its own detour at an appropriate point.

Following this, we introduce the concepts of "policy space" and "ap-

Received by the editors November 12, 1954. 
proximation in policy space," and show how they lead to monotone convergence of successive approximations to the solution of (1).

In the next solution we consider the effect upon the solution of (1) of a perturbation in the "forcing function" $g(p, q)$. This is a stability theorem; cf. $[4 ; 5]$.

We then discuss a number of possible extensions and generalizations of (1), which may be treated by means of precisely the same techniques. In passing we note a generalized equation obtained from the calculus of variations, viewed as a continuous decision process.

Following this we turn to an equation not belonging to either of the general classes treated above. Here the technique of monotone convergence plays a vital rôle in establishing the existence of a solution. The uniqueness proof is, however, of a higher level of difficulty.

Finally, in the concluding two sections, we treat the functional equation obtained from "games of survival," cf. [2], as another illustration of the analysis of equations not of types one or two. Unpublished treatments are in $[16 ; 17]$.

2. A fundamental inequality. Let us consider the two functional transformations

$$
\begin{aligned}
& \text { (a) } T_{1}(f, q)=g(p, q)+\int_{r \in D} f(r) d G(p, q, r), \\
& \text { (b) } T_{2}(f, q)=h(p, q)+\int_{r \in D} f(r) d G(p, q, r),
\end{aligned}
$$

where $d G(p, q, r) \geqq 0$, and define two additional transformations as follows:

$$
\begin{aligned}
& \text { (a) } f_{2}(p)=\operatorname{Sup}_{a} T_{1}\left(f_{1}, q\right), \\
& \text { (b) } F_{2}(p)=\operatorname{Sup}_{a} T_{2}\left(F_{1}, q\right) .
\end{aligned}
$$

The inequality we wish to prove is

LEMMA 1.

$$
\begin{aligned}
\mid f_{2}(p)- & F_{2}(p) \mid \\
& \leqq \operatorname{Sup}_{q}\left[|g(p ; q)-h(p, q)|+\int_{r \in D}\left|f_{1}(r)-F_{2}(r)\right| d G(p, q, r)\right] .
\end{aligned}
$$

Proof. Let us simplify the notation initially by assuming that both transformations in (2) have the property that the supremum is actually a maximum. Let then $q=q(p)$ be a value of $q$ for which the maximum is assumed in (2a), and $\bar{q}=\bar{q}(p)$ be a value of $\bar{q}$ for which the maximum is assumed in (2b). Then we have the following set of equalities and inequalities: 


$$
\begin{aligned}
& \text { (a) } f_{2}(p)=T_{1}\left(f_{1}, q\right) \geqq T_{1}\left(f_{1}, \bar{q}\right), \\
& \text { (b) } F_{2}(p)=T_{2}\left(F_{1}, \bar{q}\right) \geqq T_{2}\left(F_{1}, q\right) .
\end{aligned}
$$

From these follow immediately

$$
\begin{aligned}
f_{2}(p)-F_{2}(p) & \geqq\{g(p, \bar{q})-h(p, \bar{q})\}+\int_{r \in D}\left(f_{1}(r)-F_{1}(r)\right) d G(p, \bar{q}, r) \\
& \leqq\{g(p, q)-h(p, q)\}+\int_{r \in D}\left(f_{1}(r)-F_{1}(r)\right) d G(p, q, r) .
\end{aligned}
$$

These, in turn, yield

$$
\begin{aligned}
& \left|f_{2}(p)-F_{2}(p)\right| \\
& \leqq \operatorname{Max}\left[\begin{array}{l}
|g(p, \bar{q})-h(p, \bar{q})|+\int_{r \in D}\left|f_{1}(r)-F_{1}(r)\right| d G(p, \bar{q}, r) \\
|g(p, q)-h(p, q)|+\int_{r \in D}\left|f_{1}(r)-F_{1}(r)\right| d G(p, q, r)
\end{array}\right]
\end{aligned}
$$

from which the result in (3) is immediate.

To obtain the result as stated in terms of the supremum it is only necessary to note that the supremum may be obtained arbitrarily closely by the value of the function for some $q=q(p)$. The argument then proceeds via a limiting procedure.

Let us now obtain an extension of (3) which is useful in the theory of twoperson multi-stage decision processes, and, in particular, in the theory of multi-stage games.

Consider the two functional transformations

$$
\begin{aligned}
& T_{1}\left(f, q, q^{\prime}\right)=\iint_{r, r^{\prime}}\left[g\left(p, p^{\prime}, q, q^{\prime}, r, r^{\prime}\right)+f\left(r, r^{\prime}\right)\right] d G\left(p, p^{\prime}, q, q^{\prime}, r, r^{\prime}\right), \\
& T_{2}\left(f, q, q^{\prime}\right)=\iint_{r, r^{\prime}}\left[h\left(p, p^{\prime}, q, q^{\prime}, r, r^{\prime}\right)+f\left(r, r^{\prime}\right)\right] d G\left(p, p^{\prime}, q, q^{\prime}, r, r^{\prime}\right),
\end{aligned}
$$

where $d G \geqq 0$, and the two additional transformations defined as follows,

$$
\begin{aligned}
& f_{2}(p)=\operatorname{Sup}_{q} \operatorname{Inf}_{q^{\prime}} T_{1}\left(f_{1}, q, q^{\prime}\right)=\operatorname{Inf}_{q^{\prime}} \operatorname{Sup}_{q} T_{1}\left(f_{1}, q, q^{\prime}\right), \\
& F_{2}(p)=\operatorname{Sup}_{q} \operatorname{Inf}_{q^{\prime}} T_{1}\left(F_{1}, q, q^{\prime}\right)=\operatorname{Inf}_{q^{\prime}} \operatorname{Sup}_{q} T_{1}\left(F_{1}, q, q^{\prime}\right) .
\end{aligned}
$$

It is assumed that Sup Inf = Inf Sup for these transformations. This will be a consequence of the von Neumann min-max theorem and its extensions by Ville, Karlin, Ky Fan, and others, in applications. The inequality we wish to prove is 
Lemma 2.

$$
\begin{aligned}
& \left|f_{2}(p)-F_{2}(p)\right| \\
& \leqq \operatorname{Sup}_{e} \operatorname{Sup}_{\mathbf{e}^{\prime}} \iint_{r, r^{\prime}}\left[|g-h|+\left|f_{1}\left(r, r^{\prime}\right)-F_{2}\left(r, r^{\prime}\right)\right|\right] d G\left(p, p^{\prime} q, q^{\prime}, r, r^{\prime}\right) .
\end{aligned}
$$

Proof. We have, using $q, q^{\prime}, \bar{q}, \bar{q}^{\prime}$ as above,

$$
\begin{aligned}
f_{2}(p)=T_{1}\left(q, q^{\prime}, f_{1}\right) & \geqq T_{1}\left(\bar{q}, q^{\prime}, f_{1}\right) \\
& \leqq T_{1}\left(q, \bar{q}^{\prime}, f_{1}\right), \\
F_{2}(p)=T_{2}\left(\bar{q}, \bar{q}^{\prime}, F_{1}\right) & \geqq T_{2}\left(q, \bar{q}^{\prime}, F_{1}\right) \\
& \leqq T_{2}\left(\bar{q}^{\prime}, q, F_{1}\right) .
\end{aligned}
$$

Hence

$$
\begin{aligned}
f_{2}(p)-F_{2}(p) & \geqq T_{1}\left(\bar{q}, q^{\prime}, f_{1}\right)-T_{2}\left(\bar{q}, q^{\prime}, F_{1}\right) \\
& \leqq T_{1}\left(q, \bar{q}^{\prime}, f_{1}\right)-T_{2}\left(q, \bar{q}^{\prime}, F_{1}\right),
\end{aligned}
$$

or

$$
\left|f_{2}(p)-F_{2}(p)\right| \leqq \operatorname{Sup}_{q, q^{\prime}}\left|T_{1}\left(q, q^{\prime}, f_{1}\right)-T_{2}\left(q, q^{\prime}, F_{1}\right)\right|:
$$

which yields (9).

3. Equations of type one. Let us now impose the following conditions upon the function entering into the equation of (1.1):

(a) $g(p, q)$ is uniformly bounded for all $q \in S$ and all $p \in D$ satisfying the restriction $\|p\| \leqq c_{1}$, where $\|p\|=\left(\sum_{i=1}^{N} p_{i}^{2}\right)^{1 / 2} . D$ contains the null-vector $p=\theta$.

(1) (b) $g(\theta, q)=0$ for all $q \in S$, where $\theta$ represents the null vector.

(c) $|h(p, q)| \leqq 1$ for all $p \in D$ and $q \in S$.

(d) $\|T(p, q)\| \leqq a\|p\|, a<1$, for all $q \in S$ and all $p \in D$.

(e) If $v(c)=\operatorname{Sup}_{\|p\| \leq c} \operatorname{Sup}_{a}|g(p, q)|$, then $\sum_{n=0}^{\infty} v\left(a^{n} c\right)<\infty$.

Equations which satisfy these assumptions are called equations of type one. In many cases, it may be more convenient, and natural, to use the norm $\|p\|=\sum_{i=1}^{N}\left|p_{i}\right|$. It will be clear from the argumentation below that the precise form of the norm is of little importance.

Our principal result concerning these equations is the following $\left.{ }^{1}\right)$ :

(1) Announced in part in [8]. 
Theorem 1. Consider the equation

$$
\begin{aligned}
f(p) & =\operatorname{Sup}_{a}[g(p, q)+h(p, q) f(T(p, q))], \quad p \neq \theta, \\
f(\theta) & =0,
\end{aligned}
$$

assumed to be of type one.

There is a unique solution of (2) which is continuous at $p=\theta$, and defined over all of $D$. follows:

This solution may be obtained as the limit of the sequence $\left\{f_{n}(p)\right\}$ defined as

$$
\begin{aligned}
\text { (a) } f_{0}(p) & =\operatorname{Sup} g(p, q), \\
\text { (b) } f_{n+1}(p) & =\operatorname{Sup}_{a}\left[g(p, q)+h(p, q) f_{n}(T(p, q))\right], \quad n=0,1,2, \cdots
\end{aligned}
$$

Alternatively, any initial function $f_{0}(p)$ which is continuous at $p=\theta$ and bounded for $\|p\| \leqq c_{1}, p \in D$, may be used in (3b) to yield a convergent sequence.

If $g(p, q), h(p, q)$, and $T(p, q)$ are continuous in any bounded position of $D$, uniformly for all $q \in S$, then $f(p)$ is continuous in any bounded portion of $D$.

Proof. Let us consider the sequence defined by (3). Using Lemma 1, proved in $\$ 2$, we have for $n \geqq 1$,

$$
\begin{aligned}
\left|f_{n+1}(p)-f_{n}(p)\right| & \leqq \operatorname{Sup}_{q} \mid h(p, q)\left(f_{n}(T(p, q))-f_{n-1}(T(p, q)) \mid\right. \\
& \leqq \operatorname{Sup}_{q}\left|f_{n}(T(p, q))-f_{n-1}(T(p, q))\right|
\end{aligned}
$$

and

$$
\left|f_{1}(p)-f_{0}(p)\right| \leqq \operatorname{Sup}_{q}\left|f_{0}(T(p, q))\right|=\operatorname{Sup}_{q}|g(p, q)| .
$$

Let us now define the new sequence

$$
v_{n}(c)=\operatorname{Sup}_{p}\left|f_{n+1}(p)-f_{n}(p)\right|, \quad\|p\| \leqq c, p \in D .
$$

Using the function defined in (1e), we see that $v_{0}(c)=v(c)$. Turning to (4) we have, for $p \in D,\|p\| \leqq c$,

$$
\begin{aligned}
\operatorname{Sup}_{p}\left|f_{n+1}(p)-f_{n}(p)\right| & \leqq \operatorname{Sup}_{p} \operatorname{Sup}_{q}\left|f_{n}(T(p, q))-f_{n-1}(T(p, q))\right| \\
& \leqq \operatorname{Sup}_{\|p\| \leq a c}\left|f_{n}(p)-f_{n-1}(p)\right|,
\end{aligned}
$$

by virtue of our assumption concerning $T(p, q)$. Hence $v_{n+1}(c) \leqq v_{n}(a c)$, $n=0,1,2, \cdots$, or $v_{n}(c) \leqq v_{0}\left(a^{n} c\right)$. It follows that the series $\sum_{n=0}^{\infty}\left[f_{n+1}(p)\right.$ $\left.-f_{n}(p)\right]$ converges uniformly for $\|p\| \leqq c$, and hence that $\left\{f_{n}(p)\right\}$ converges 
uniformly to a function $f(p)$ for $\|p\| \leqq c$.

This completes the proof of existence and the proof of the statements concerning convergence and continuity.

To establish uniqueness, let $f(p)$ and $F(p)$ be two solutions of (1) continuous at $p=\theta$, and hence defined for all $p \in D$. Let

$$
v(c)=\operatorname{Sup}_{p}|f(p)-F(p)|, \quad\|p\| \neq c, p \in D .
$$

Applying Lemma 1, we have

$$
|f(p)-F(p)| \leqq \operatorname{Sup}_{q}[f(T(p, q))-F(T(p, q))]
$$

whence

$$
v(c) \leqq v(a c) \leqq \cdots \leqq v\left(a^{n} c\right) .
$$

Since $f(p)$ and $F(p)$ are continuous for $p=0, v\left(a^{n} c\right) \rightarrow 0$ as $n \rightarrow \infty$. Hence $v(c) \equiv 0$, and $f(p) \equiv F(p)$.

4. A multi-stage decision process of deterministic type yielding an equation of type one. Let us consider the following multi-stage decision process. We start with an initial vector $p$ and choose a transformation $T(p, q)$, for $q \in S$. As a result of this choice, $p$ is transformed into $T(p, q)$ and we obtain a return $g(p, q)$. The process is now repeated ad infinitum and it is required to determine the sequence of choices which maximizes the total return. If we call $f(p)$ this maximum return, we obtain equation (3.1) for $f(p)$.

An interesting example of the above equation is

$$
f(x)=\underset{0 \leq y \leq x}{\operatorname{Max}}[g(y)+h(x-y)+f(a y+b(x-y))], \quad f(0)=0,
$$

where $0<a, b<1$, which occurs in the theory of multi-stage allocation and investment problems.

A good deal of information concerning the form of the solution can be obtained for various special classes of functions, in particular, if
(a) $g$ and $h$ are both convex,
(b) $g$ and $h$ are both concave,
(c) $g(x)=c x^{d}, h(x)=e x^{f}$,
(d) $g$ and $h$ are both quadratic.

See $[2 ; 7 ; 9 ; 10 ; 11]$.

5. Equations of type two. Let us now consider the equation of (1.1) where we impose the conditions
(a) $|g(p, q)|$ is uniformly bounded for all $q \in S$, and $\|p\| \leqq c_{1}, p \in D$.
(b) $|h(p, q)| \leqq a<1$ for all $q \in S$ and uniformly in any region $\|p\| \leqq c_{1}$, $p \in D$.
(c) $\|T(p, q)\| \leqq\|p\|$, or $D$ is a bounded region. 
Equations satisfying these conditions we shall call equations of type two. We shall demonstrate.

TheOREM 2. If

$$
f(p)=\operatorname{Sup}_{q}[g(p, q)+h(p, q) f(T(p, q))]
$$

is an equation of type two, there is a unique solution which is bounded in any finite part of $D$.

The solution may be found by means of successive approximations as before, and the previous statements concerning continuity of the solution remain valid.

Proof. Let

$$
\begin{aligned}
f_{0}(p) & =\operatorname{Sup}_{q} g(p, q), \\
f_{n+1}(p) & =\operatorname{Sup}_{q}\left[g(p, q)+h(p, q) f_{n}(T(p, q))\right], \quad n=0,1,2, \cdots .
\end{aligned}
$$

Using Lemma 1, we have

$$
\begin{aligned}
\left|f_{n+1}(p)-f_{n}(p)\right| & \leqq \operatorname{Sup}_{q}\left|h(p, q)\left[f_{n}(T(p, q))-f_{n-1}(T(p, q))\right]\right| \\
& \leqq a \operatorname{Sup}_{q}\left|f_{n}(T(p, q))-f_{n-1}(T(p, q))\right|,
\end{aligned}
$$

where $a<1$. From this point on the proof clearly parallels the proof of Theorem 1 .

6. A multi-stage decision process of stochastic type which yields an equation of type two. Let us consider the following multi-stage decision process of stochastic type. At some initial time we have a physical system in a state given by the vector $p$, and have a choice of a set of transformations $\{T(p, q)\}$ which will convert $p$ into $T(p, q)$ and yield a return of $R(p, q)$ with probability $h(p, q)$, and with probability $1-h(p, q)$ terminate the process. The problem is that of determining the sequence of choices which maximizes the expected return obtained before the process terminates. tion

$$
f(p)=\operatorname{Sup}_{e}[h(p, q)\{R(p, q)+f(T(p, q))\}] .
$$

An interesting example, which has been discussed in several places, [2; 3; $6 ; 11]$, is

$$
f(x, y)=\operatorname{Max}\left[\begin{array}{l}
p_{1}\left[r_{1} x+f\left(\left(1-r_{1}\right) x, y\right)\right] \\
q_{1}\left[s_{1} y+f\left(x,\left(1-s_{1}\right) y\right)\right]
\end{array}\right], \quad x, y>0
$$

where $0<p_{1}, q_{1}, r_{1}, s_{1}<1$. 
Continuous versions are discussed in $[18 ; 19]$.

7. Monotone convergence. We have in the preceding sections demonstrated convergence of the successive approximation under assumptions which yielded essentially geometric convergence. Let us now show that, under the assumption that $h(p, q) \geqq 0$, which is true in all the applications to date, we have at our disposal a method of choosing an initial approximation which will yield monotone convergence.

Let us consider our equation in the form

$$
f(p)=\underset{q}{\operatorname{Max}}[g(p, q)+h(p, q) f(T(p, q))] .
$$

There are now two functions of particular interest. There is first of all the function $f(p)$ itself, and secondly the function $g=q(p)$ which yields the maximum in (1). Let us agree to call any function $q(p)$ which is admissible a policy, and a function which yields the maximum, an optimal policy.

It is clear that any particular optimal policy determines $f(p)$ iteratively, and conversely a knowledge of $f(p)$ determines all optimal policies, $q(p)$. We thus have, side by side, two function spaces $\{f(p)\}$ and $\{q(p)\}$; the former will be called function space and the latter policy space.

In view of this duality between function space and policy space, we have the privilege of determining the solution of (1) in one or the other spaces and thus of choosing the most convenient space. In many ways, the determination in policy space is a more natural, more informative, and simpler description of the solution. In particular, we shall show that an initial approximation in policy space always yields monotone convergence in function space.

Let $q_{0}=q_{0}(p)$ be an initial approximation to $q(p)$ and let $f_{0}(p)$ be determined by use of this policy, i.e.,

$$
f_{0}(p)=g\left(p, q_{0}\right)+h\left(p, q_{0}\right) f_{0}\left(T\left(p, q_{0}\right)\right),
$$

and the sequence $\left\{f_{n}(p)\right\}, n=1,2, \cdots$, then be determined recursively,

$$
f_{n+1}(p)=\operatorname{Sup}_{q}\left[g(p, q)+h(p, q) f_{n}(T(p, q))\right], n=0,1,2, \cdots
$$

[Having introduced the concept of approximation in policy space, it is now convenient to use the supremum again to bypass questions of continuity over $q$.] Let us assume, as in the case of equations of types one and two, that sufficient conditions have been imposed to have the sequence $\left\{f_{n}(p)\right\}$ uniformly bounded in any finite portion of $D$.

It is immediately seen that $f_{1}(p) \geqq f_{0}(p)$, and therefore, by virtue of the non-negativity of $h(p, q)$, that $f_{n+1}(p) \geqq f_{n}(p)$ for all $n$. It follows that $f_{n}(p)$ converges to $f(p)$ as $n \rightarrow \infty$, in any finite part of $D$.

If $q$ is a member of a finite set $S$, there is no question of the convergence of $\left\{f_{n}(p)\right\}$ to an actual solution of $(3)$, where the supremum is now a maximum. If $S$ contains a continuum, it is not immediate that $f(p)$ is a solution of 


$$
f(p)=\operatorname{Sup}_{a}[g(p, q)+h(p, q) f(T(p, q))] .
$$

To establish this, we observe that by virtue of the monotone convergence,

$$
f_{n+1}(p) \leqq \operatorname{Sup}_{q}[g(p, q)+h(p, q) f(T(p, q))]
$$

whence

$$
f(p) \leqq \operatorname{Sup}_{q}[g(p, q)+h(p, q) f(T(p, q))] .
$$

On the other hand, we have

$$
\begin{aligned}
f(p) & =\operatorname{Sup}_{q}\left[g(p, q)+h(p, q) f_{n}(T(p, q))\right] \\
& \geqq g(p, q)+h(p, q) f_{n}(T(p, q))
\end{aligned}
$$

for all $q \in S$ and all $n$. Letting $n \rightarrow \infty$, we obtain the reverse inequality to (6), and hence equality.

This property of monotone convergence is particularly important in other parts of the theory of dynamic programming, in particular, in applications to the calculus of variations; $\mathrm{cf} .[3 ; 13 ; 15]$.

8. Stability theorems. In the theory of functional equations a problem of great theoretical interest, with important physical ramifications, is that of the dependence of the solution upon the form of the equation. It is of much interest to determine those equations which have the property that small changes in the form of the equation effect correspondingly small changes in the form of the solution. For a discussion with reference to differential and difference equations, see $[4 ; 5]$.

Let us now consider the two equations,

$$
\begin{aligned}
& \text { (a) } f(p)=\operatorname{Sup}_{e}[g(p, q)+h(p, q) f(T(p, q))] \text {, } \\
& \text { (b) } F(p)=\operatorname{Sup}_{q}[G(p, q)+h(p, q) F(T(p, q))] \text {, }
\end{aligned}
$$

and assume, to begin with, that they are both of type one. We wish to obtain an inequality $\operatorname{Sup}_{p}|f(p)-F(p)|, p \in D,\|p\| \leqq c$.

To obtain this inequality, we employ the method of successive approximations in both equations, setting

$$
\begin{aligned}
f_{1}(p) & =\operatorname{Sup}_{q} g(p, q), \\
f_{n+1}(p) & =\operatorname{Sup}_{q}\left[g(p, q)+h(p, q) f_{n}(T(p, q))\right], \\
F_{1}(p) & =\operatorname{Sup}_{q} G(p, q), \\
F_{n+1}(p) & =\operatorname{Sup}_{p}\left[G(p, q)+h(p, q) F_{n}(T(p, q))\right] .
\end{aligned}
$$


We have

$$
\left|F_{1}(p)-f_{1}(p)\right| \leqq \operatorname{Sup}_{q}|G(p, q)-g(p, q)|
$$

and

$$
\begin{aligned}
\left|F_{n+1}(p)-\quad .(p)\right| \leqq & \operatorname{Sup}_{a}[|G(p, q)-g(p, q)| \\
& \left.+|h(p, q)|\left|F_{n}(T(p, q))-f_{n}(T(p, q))\right|\right] .
\end{aligned}
$$

Let us define

$$
u(c)=\operatorname{Sup}_{\|p\| \leqq c} \operatorname{Sup}_{q}|G(p, q)-g(p, q)|
$$

Then we have

THEOREM 3. With the above notation, for equations of type one,

$$
\operatorname{Sup}_{\|p\| \leqq c}|F(p)-f(p)| \leqq \sum_{n=0}^{\infty} u\left(a^{n} c\right) .
$$

Proof. Set

$$
w_{n}(c)=\operatorname{Sup}_{\|\neq\| \leqq c}\left|F_{n}(p)-f_{n}(p)\right| .
$$

Let us show inductively that we have $w_{n}(c) \leqq \sum_{k=0}^{n-1} u\left(a^{k} c\right), n \geqq 1$. Using (4), and the hypotheses governing an equation of type one, this is easily seen. Letting $n \rightarrow \infty$, we obtain (6).

Similarly,

THEOREM 4. With the above notation, for equations of type two,

$$
\operatorname{Sup}_{\|p\| \leqq c} F|(p)-f(p)| \leqq u(c) /(1-a) .
$$

The proof follows the same lines as above and is therefore omitted.

Similar estimates can be obtained in the cases where $h(p, q)$ and $T(p, q)$ are perturbed.

9. Some directions of generalization. A first generalization of (1.1) is the equation

$$
f(p)=\operatorname{Sup}_{q}\left[g(p, q)+\sum_{i=1}^{N} h_{i}(p, q) f\left(T_{i}(p, q)\right)\right],
$$

which, in turn, is a particular case of

$$
f(p)=\operatorname{Sup}_{q}\left[g(p, q)+\int_{r \in D} f(r) d G(p, q, r)\right]
$$

The methods utilized above yield analogues of the preceding theorems for 
the above equations, and for the system

$$
f_{i}(p)=\operatorname{Sup}_{q}\left[g_{i}(p, q)+\sum_{j=1}^{N} \int_{r \in D} f_{j}(r) d G_{i j}(p, q, r)\right], \quad i=1,2, \cdots, N .
$$

An example of (2) is the equation of "optimal inventory,"

$$
f(x)=\operatorname{Inf}_{y \geq x}\left[v(x, y)+\alpha\left[(1-G(y)) f(0)+\int_{0}^{\nu} f(y-s) d G(s)\right]\right],
$$

discussed first by Arrow, Harris, and Marschak [1], and then more extensively by Dvoretzky, Kiefer, and Wolfowitz [20]; see also [12], for some particular cases.

Similarly, using Lemma 2, we may derive analogous theorems for the functional equation

(5)

$$
\begin{aligned}
f\left(p, p^{\prime}\right)= & \operatorname{Sup}_{\boldsymbol{q}} \operatorname{Inf}_{q^{\prime}}\left[\int \int _ { r , r ^ { \prime } \in D } \left[g\left(p, p^{\prime}, q, q^{\prime}, r, r^{\prime}\right)\right.\right. \\
& \left.\left.+f\left(r, r^{\prime}\right)\right] d G\left(p, p^{\prime}, q, q^{\prime}, r, r^{\prime}\right)\right] \\
= & \operatorname{Inf}_{q^{\prime}} \operatorname{Sup}_{q}\left[\int \int _ { r , r ^ { \prime } \in D } \left[g\left(p, p^{\prime}, q, q^{\prime}, r, r^{\prime}\right)\right.\right. \\
& \left.\left.+f\left(r, r^{\prime}\right)\right] d G\left(p, p^{\prime}, q, q^{\prime}, r, r^{\prime}\right)\right],
\end{aligned}
$$

an equation occurring in the theory of multi-stage games, as we shall discuss below. See [11].

Finally let us mention in passing that many problems in the calculus of variations may be converted into problems involving functional equations having a form analogous to (1). Consider, for example, the problem of maximizing $J(y)=\int_{0}^{T} F\left(y, y^{\prime}, t\right) d t$, or more generally the problem of maximizing

$$
J(y)=\int_{a}^{T} F(x, y, t) d t
$$

given

$$
d x / d t=G(x, y, t), \quad x(a)=c .
$$

Assuming that the maximum exists, we set

$$
\operatorname{Max}_{y} J(y)=f(a, c) .
$$

It is readily seen that $f(a, c)$ satisfies the functional equation 
(9) $f(a, c)=\operatorname{Max}_{y[a, a+s]}\left[\int_{a}^{a+s} F(x, y, t) d t+f\left(a+s, c+\int_{a}^{a+s} G(x, y, t) d t\right)\right]$.

In this way we obtain analogues of (1.1) involving maximization over function spaces of functional transformations. The reader who is interested in the above discussion of problems in the calculus of variations as multi-stage decision problems of continuous type may refer to $[2 ; 11 ; 13 ; 15]$, where the nonlinear partial differential equation corresponding to (9) is discussed for the above case, and for eigenvalue problems.

Analogous results may be obtained for partial differential equations, variational problems involving translation operators, and so on. These results will be presented subsequently.

10. An equation of the third type. The technique of approximation in policy space which yields monotone convergence, discussed above in $\S 7$, is very useful in establishing the existence of solutions of equations of type three, a class defined quite simply as the complementary class of equations of type one and type two.

The uniqueness of the solution of equations of type three is in general a problem of a greater level of difficulty, as we shall see below and in $\$ 13$.

Let us illustrate these remarks by considering the functional equation

$$
\begin{aligned}
f(p) & =\operatorname{Min}\left[1+\sum_{k=0}^{n} p_{k} f\left(x_{k}\right), \operatorname{Min}_{l}\left[1+f\left(T_{l} p\right)\right]\right], \quad p \neq x_{0}, \\
f\left(x_{0}\right) & =0
\end{aligned}
$$

where $l$ runs over the set of integers $1,2, \cdots, M$. Here we set

$$
p=\left(p_{0}, p_{1}, \cdots, p_{n}\right), \quad p_{i} \geqq 0, \sum_{i=0}^{n} p_{i}=1,
$$

$$
\begin{array}{r}
T_{l} p=\left(p_{0 l}, p_{1 l}, \cdots, p_{n l}\right), \quad p_{i l} \geqq 0, p_{0 l} \neq 1, \sum_{i=0}^{n} p_{i l}=1, \\
l=1,2, \cdots, M,
\end{array}
$$

$x_{k}=(0, \cdots, 1, \cdots, 0)$, the one occurring in the $k$ th place. The function $f(p)$ is a scalar function of $p$.

We shall prove

THEOREM 5. If for each transformation $T_{l}$ it is true that

$$
\sum_{k=1}^{n} p_{k l} \leqq c_{1}, \quad 0<c_{1}<1,
$$

then there exists a unique bounded positive solution to (1) above. This function is positive for $p \neq x$. 
Proof. We shall employ the method of successive approximations, using as our first approximation an approximation in policy space. Let us represent by $L$ the choice of $1+\sum_{k=0}^{n} p_{k} f\left(x_{k}\right)$, and by $T_{1}$ the choice of $l=1$ in (1). We consider the function $F_{1}(p)$ determined by the policy symbolized by $L T_{1} L T_{1} \cdots$, and the function $F_{2}(p)$ determined by the policy $T_{1} L T_{1} L \cdots$. It is clear that

$$
\begin{array}{ll}
F_{1}(p)=1+F_{2}\left(T_{1} p\right), & p \neq x_{0}, \\
F_{2}(p)=1+\sum_{k=0}^{n} p_{k l} F_{1}\left(x_{k}\right), & p \neq x_{0}, \\
F_{1}\left(x_{0}\right)=F_{2}\left(x_{0}\right)=0 . &
\end{array}
$$

Hence,

$$
F_{1}\left(x_{l}\right)=2+\sum_{k=1}^{n} p_{k l} F_{1}\left(x_{k}\right), \quad l=1,2, \cdots, n .
$$

Since, by assumptions, $\sum_{k=0}^{n} p_{k l}<c_{1}$, the determinant of the system does not vanish and the system has a unique solution, necessarily positive, as we see by solving iteratively. Having determined $F_{1}\left(x_{\imath}\right)$, the determination of $F_{2}(p)$ and, hence, $F_{1}(p)$ for general $p$, is immediate.

To begin our successive approximations, define $\left({ }^{2}\right)$

$$
f_{0}(p)=\operatorname{Min}\left[F_{1}(p), F_{2}(p)\right]
$$

(6)

$$
\begin{aligned}
& f_{n+1}(p)=\operatorname{Min}\left[\left[1+\sum_{k=1}^{n} p_{k} f_{n}\left(x_{k}\right)\right], \operatorname{Min}_{\imath}\left[1+f_{n}\left(T_{l} p\right)\right]\right], \quad p \neq x_{0}, \\
& f_{n+1}\left(x_{0}\right)=0 .
\end{aligned}
$$

It is readily seen that $f_{0}(p) \geqq f_{1}(p) \geqq \cdots \geqq f_{n}(p) \geqq 1, p \neq x_{0}$.

Hence $f_{n}(p)$ converges monotonically to a function $f(p)$ which clearly satisfies the functional equation. This establishes the existence of a bounded solution.

The uniqueness proof is considerably more complicated and proceeds in a series of steps. Let $f(p)$ and $g(p)$ be two bounded solutions of (1). The first step is

Lemma 2. $\operatorname{Sup}_{p}|f(p)-g(p)|=\operatorname{Max}_{k}\left|f\left(x_{k}\right)-g\left(x_{k}\right)\right|$.

Proof. The inequality

$$
\operatorname{Max}_{k}\left|f\left(x_{k}\right)-g\left(x_{k}\right)\right| \leqq \operatorname{Sup}_{p}|f(p)-g(p)|
$$

is clear. To demonstrate the reverse inequality, we consider four cases:

(2) I owe this felicitous choice of an initial approximation to H. N. Shapiro. 
(8)
(a) $f(p)=1+\sum_{k=1}^{n} p_{k} f\left(x_{k}\right)$,
$g(p)=1+\sum_{k=1}^{n} p_{k} g\left(x_{k}\right)$
(b) $f(p)=1+\sum_{k=1}^{n} p_{k} f\left(x_{k}\right)$,
$g(p)=1+g\left(T_{\imath} p\right)$

(c) $f(p)=1+f\left(T_{\imath} p\right)$,

$g(p)=1+\sum_{k=1}^{n} p_{k} g\left(x_{k}\right)$

(d) $f(p)=1+f\left(T_{\imath} p\right)$,

$g(p)=1+g\left(T_{l} p\right)$.

Consider first the case corresponding to (a). We have

$$
f(p)-g(p)=\sum_{k=0}^{n} p_{k}\left[f\left(x_{k}\right)-g\left(x_{k}\right)\right]
$$

whence

$$
|f(p)-g(p)| \leqq \operatorname{Max}_{k}\left|f\left(x_{k}\right)-g\left(x_{k}\right)\right|
$$

Therefore for all $p$ for which (8a) holds, the lemma is correct. Equation (8a) will hold whenever $p$ is close to $x_{0}$, since $1+\sum_{k=1}^{n} p_{k} f\left(x_{k}\right)$ is less than 2 in this case, and $1+f\left(T_{\imath} p\right) \geqq 2$. Thus $1+f\left(T_{\imath} p\right)$ and $1+g\left(T_{\imath} p\right)$ will for $l=1,2, \cdots, M$ exceed the result of the $L$-move for $p$ close to $x_{0}$.

This is an important point since the crux of our proof is the fact that (8a) will always occur after a finite number of moves, by virtue of the condition in (3).

Now consider case $(8 \mathrm{~b})$. We have

$$
\begin{aligned}
& f(p)=1+\sum_{k=0}^{n} p_{k} f\left(x_{k}\right) \leqq 1+f\left(T_{l} p\right), \\
& g(p)=1+g\left(T_{l} p\right) \leqq 1+\sum_{k=0}^{n} p_{k} g\left(x_{k}\right) .
\end{aligned}
$$

Hence

(12) $|f(p)-g(p)| \leqq \operatorname{Max}\left\{\underset{k}{\operatorname{Max}}\left|f\left(x_{k}\right)-g\left(x_{k}\right)\right|, \operatorname{Sup}_{p}\left|f\left(T_{\imath} p\right)-g\left(T_{\imath} p\right)\right|\right\}$ and similarly for (8c).

From (8d) we derive

$$
|f(p)-g(p)| \leqq \operatorname{Max}\left\{\left|f\left(T_{\imath} p\right)-g\left(T_{\imath} p\right)\right|,\left|f\left(T_{l^{\prime}} p\right)-g\left(T_{l^{\prime}} p\right)\right|\right\} .
$$

We now iterate these inequalities. For any fixed $p, T_{l_{1}} T_{l_{2}} \cdots T_{l_{n}} p$ will be in the region governed by ( $8 \mathrm{a})$ for $n$ large enough. Consequently, we obtain 


$$
\operatorname{Sup}_{p}|f(p)-g(p)| \leqq \operatorname{Max}_{k}\left|f\left(x_{k}\right)-g\left(x_{k}\right)\right| .
$$

This completes the proof of the lemma.

It remains to show that $\operatorname{Max}_{k}\left|f\left(x_{k}\right)-g\left(x_{k}\right)\right|=0$. Let $K$ be an index at which the maximum is assumed. It follows from the functional equation for $f$ and $g$ that

$$
\begin{aligned}
f\left(x_{k}\right) & =1+f\left(T_{l} x_{k}\right), & l & =l(k), \\
g\left(x_{k}\right) & =1+g\left(T_{l^{\prime}} x_{k}\right), & l^{\prime} & =l^{\prime}(k) .
\end{aligned}
$$

As above we have

$$
\begin{aligned}
& f\left(x_{k}\right)=1+f\left(T_{l} x_{k}\right) \leqq 1+f\left(T_{l}{ }^{\prime} x_{k}\right), \\
& g\left(x_{k}\right)=1+g\left(T_{l} x_{k}\right) \leqq 1+g\left(T_{l} x_{k}\right) .
\end{aligned}
$$

If both inequalities are proper we have

$$
\begin{aligned}
\left|f\left(x_{k}\right)-g\left(x_{k}\right)\right| & <\operatorname{Max}|| f\left(T_{l} x_{k}\right)-g\left(T_{\imath} x_{k}\right)|,| f\left(T_{l^{\prime}} x_{k}\right)-g\left(T_{l^{\prime}} x_{k}\right)|| \\
& <\operatorname{Sup}_{p}|f(p)-g(p)|
\end{aligned}
$$

a contradiction.

Thus for either $l$ or $l^{\prime}$ we have

$$
f\left(x_{k}\right)=1+f\left(T_{l} x_{k}\right), \quad g\left(x_{k}\right)=1+g\left(T_{l} x_{k}\right) .
$$

This means that the first choices from the position $x_{k}$ can be the same.

Consider now the situation for second moves. Using the same argument, we see that the second moves, i.e., the equations for $f\left(T_{l} x_{k}\right)$ and $g\left(T_{l} x_{k}\right)$, can also be the same, and so on, inductively.

Let $p_{n}=p_{n}\left(x_{k}\right)$ be the distribution achieved after $n$ moves, where the $(n+1)$ st move puts $x_{k}$ into the region governed by (8a). The argument above shows that $f$ and $g$ land in this region on the same move. Thus,

$$
\begin{aligned}
& f\left(x_{k}\right)=(n+1)+\sum_{k=0}^{n} p_{k n} f\left(x_{k}\right), \\
& g\left(x_{k}\right)=(n+1)+\sum_{k=0}^{n} p_{k n} g\left(x_{k}\right),
\end{aligned}
$$

and consequently

$$
\begin{aligned}
\left|f\left(x_{k}\right)-g\left(x_{k}\right)\right| & \leqq \sum_{k=1}^{n}\left|f\left(x_{k}\right)-g\left(x_{k}\right)\right| \\
& \leqq\left|1-p_{0 n}\right| \operatorname{Sup}_{q}\left|f\left(x_{k}\right)-g\left(x_{k}\right)\right| .
\end{aligned}
$$

Since $1>p_{0 n}>0$, this implies that $\left|f\left(x_{k}\right)-g\left(x_{k}\right)\right|=0$. Hence $\operatorname{Sup}_{p}|f(p)-g(p)|$ $=0$, which completes our uniqueness proof. 
11. A multi-stage decision process yielding an equation of the third type. Let us consider a physical system which at any time may be in one of the $(n+1)$ states specified by the vectors $x_{k}, k=0,1,2, \cdots, n$. Let $p=\left(p_{0}\right.$, $\left.p_{1}, \cdots, p_{n}\right)$ be a probability distribution specifying the probability $p_{k}$ that the system is in the $k$ th state.

It is desired to have the system in the 0th state. At any time we may look at the system and determine the precise state of the system, or we may effect a transformation upon the system which transforms $p$ into $T_{\imath} p, l=1,2, \cdots, M$, where $T_{l}$ has the properties described above. Each of these operations consumes a unit time. The problem is that of determining the policy which minimizes the expected time required to transform the system into state 0 with certainty. If $f(p)$ is the minimum expected time required starting from the a priori distribution $p$, then $f(p)$ satisfies (11.1).

12. Games of survival. Generalities. The theory of two-person multistage games yields a number of equations having the properties of equations of types 1,2 , or 3 . A particular class of games of survival yields a functional equation which has many interesting and difficult features.

Let us consider a two-person zero-sum game specified by the game matrix

$$
A=\left(a_{i j}\right), \quad i, j=1,2, \cdots, n,
$$

and assume that the two players $P$ and $Q$ start initially with 0 quantities $x$ and $y$. Since the game is zero-sum the state of the game is specified at any time by the quantity possessed by $A, x$. Let $x+y=d$, a fixed quantity.

We now assume that both players, instead of playing to maximize their respective expected gains in each play, play to ruin their opponent. This is a generalized "gambler's ruin" process, a particular type of Markoff process, in which each player has some control over his transition probabilities.

Let us then define for $0<x<d$

(2) $f(x)=$ the probability that $Q$ is ruined before $P$ when $P$ has $x$ and both players use optimal play,

and set $f(x)=0, x \leqq 0, f(x)=1, x \geqq d$.

Assuming that this function $f(x)$ exists, it satisfies the equation

$$
\begin{aligned}
f(x) & =\underset{Q}{\operatorname{Min}_{\boldsymbol{p}}} \operatorname{Max}_{p}\left[\sum_{i, j=1}^{N} p_{i} q_{j} f\left(x+a_{i j}\right)\right] \\
& =\underset{p}{\operatorname{Max}_{Q}} \operatorname{Min}_{Q}\left[\sum_{i, j=1}^{N} p_{i} q_{j} f\left(x+a_{i j}\right)\right] .
\end{aligned}
$$

This follows the formulation originally given in [17]; cf. also [2].

Similarly, if we consider a non-zero sum game where $P$ and $Q$ have respectively the game matrices 


$$
A=\left(a_{i j}\right), \quad B=\left(b_{i j}\right),
$$$$
a_{i j}+b_{i j} \leqq 0,
$$

we must now define

(5) $f(x, y)=$ the probability that $Q$ is ruined before $P$ when $P$ has $x, Q$ has $y$, and both players employ optimal play.

This function satisfies the equation

$$
\begin{array}{rlr}
f(x, y) & =\underset{q}{\operatorname{Min}} \operatorname{Max}_{p}\left[\sum_{i, j=1}^{N} p_{i} q_{j} f\left(x+a_{i j}, y+b_{i j}\right)\right] \\
& =\underset{p}{\operatorname{Max}_{q}} \operatorname{Min}_{q}\left[\sum_{i, j=1}^{N} p_{i} q_{j} f\left(x+a_{i j}, y+b_{i j}\right)\right], & x, y>0, \\
f(x, y)=0, & x \leqq 0, y>0, \\
f(x, y)=1, & x>0, y \leqq 0, \\
f(0,0)=0, & \text { (a matter of convention). }
\end{array}
$$

This last equation is of type 1 if $a_{i j}+b_{i j}<0$, and correspondingly fairly simple to discuss. The more difficult equation is (3). In the following section we shall prove an existence and uniqueness theorem for a particular case for this equation.

13. Games of survival. Existence and uniqueness. We shall prove the following result:

Theorem 6. Consider the equation

$$
\begin{aligned}
f(x) & =\underset{q}{\operatorname{Min}} \operatorname{Max}_{p}\left[p_{1} q_{1} f(x-1)+p_{1} q_{2} f(x+a)+p_{2} q_{1} f(x+c)+p_{2} q_{2} f(x-b)\right] \\
& =\operatorname{Max}_{p} \operatorname{Min}_{q}\left[p_{1} q_{1} f(x-1)+p_{1} q_{2} f(x+a)+p_{2} q_{1} f(x+c)+p_{2} q_{2} f(x-b)\right],
\end{aligned}
$$

for $x=1,2,3, \cdots, d-1$, associated with the game matrix

$$
A=\left(\begin{array}{rr}
-1 & a \\
c & -b
\end{array}\right),
$$

where $a, b$, and $c$ are positive integers, with

$$
f(x)=0, \quad x \leqq 0, \quad f(x)=1, \quad x \geqq d .
$$

There is a unique function $f(x)$ satisfying the inequalities $0 \leqq f(x) \leqq 1$, which satisfies (1) and (3).

Proof. To simplify the notation, let us set $V(f(x))$ as the value of the game whose matrix is

$$
\left(\begin{array}{ll}
f(x-1) & f(x+a) \\
f(x+c) & f(x-b)
\end{array}\right)
$$


The functional equation in (1) has the form

$$
\begin{array}{rlrl}
f(x) & =V(f(x)), & x=1,2, \cdots, & d-1, \\
f(x) & =0, & x \leqq 0, \\
f(x) & =1, & x \geqq d .
\end{array}
$$

Let us define the sequence $\left\{f_{n}(x)\right\}$ as follows.

$$
\begin{aligned}
f_{0}(x) & = \begin{cases}1, & x \geqq d, \\
0, & x \leqq d-1,\end{cases} \\
f_{n+1}(x) & =V\left(f_{n}(x)\right), \\
f_{n+1}(x) & = \begin{cases}1, & x=0,1,2, \cdots, x=1,2, \cdots, d-1, \\
0, & x \leqq 0 .\end{cases}
\end{aligned}
$$

It is clear that $f_{1}(x) \geqq f_{0}(x)$ for all $x$, and hence inductively that $f_{n+1}(x)$ $\geqq f_{n}(x)$. It follows from the fact that $0 \leqq f_{n}(x) \leqq 1$ for all $x$ and $n$ that $f_{n}(x)$ converges as $n \rightarrow \infty$ for all $x$ to a function $f(x)$. That $f(x)$ satisfies (5) is a consequence of the continuity of the value of a game as a continuous function of the game matrix. This completes the proof of existence.

Since $f_{0}(x)$ is a monotone increasing function of $x$, each function $f_{n}(x)$ is monotone increasing, and hence $f(x)$ is monotone increasing. Let us now demonstrate the important result that it is actually strictly monotone. Upon this fact the proof of uniqueness depends.

We have

$$
f(1)=V\left(\begin{array}{cc}
0 & f(a) \\
f(c) & 0
\end{array}\right)
$$

If $f(a)$ and $f(c)$ are positive, we have $f(1)>0$.

To establish this, let us assume, on the contrary, that $f(x)=0$, for $x=0$, $1,2, \cdots, k<d$, but $f(k+1) \neq 0$. Then

$$
f(k)=V\left(\begin{array}{ll}
f(k-1) & f(k+a) \\
f(k+c) & f(k-b)
\end{array}\right)=V\left(\begin{array}{cc}
0 & f(k+a) \\
f(k+c) & 0
\end{array}\right) .
$$

Since $f(k+a) \geqq f(k+1)>0, f(k+c) \geqq f(k+1)>0$, it follows that $f(k)>0$, which is a contradiction unless $k=0$. Thus $f(1)>0$.

We have

$$
f(2)=V\left(\begin{array}{cc}
f(1) & f(a+2) \\
f(c+2) & f(2-b)
\end{array}\right) .
$$

Since $f(1)>0, f(a+2) \geqq f(a+1), f(c+2) \geqq f(c+2), f(2-b) \geqq 0$, we must have $f(2)>f(1)$, unless $f(2-b)=0$ and the solution is $p_{2}=q_{2}=1$. This is clearly impossible since it yields $f(2)=0<f(1)$ and we know that $f(2) \geqq f(1)$. 
We thus prove, inductively, that

$$
0 \leqq f(0)<f(1)<f(2)<\cdots<f(d)=1,
$$

with strict inequality at every step.

The uniqueness now follows readily. Let us set

$$
T(p, q, f)=p_{1} q_{1} f(x-1)+p_{1} q_{2} f(x+a)+p_{2} q_{1} f(x+c)+p_{2} q_{2} f(x-b) .
$$

Let $f$ and $g$ be solutions of

$$
\begin{aligned}
& f(x)=\underset{q}{\operatorname{Min}} \operatorname{Max}_{p} T(p, q, f)=\underset{p}{\operatorname{Max}} \underset{q}{\operatorname{Min}} T(p, q, f), \\
& g(x)=\operatorname{Min}_{q} \operatorname{Max}_{p} T(p, q, g)=\underset{p}{\operatorname{Max}} \operatorname{Min}_{q} T(p, q, g),
\end{aligned}
$$

for $0<x<d$, and

$$
f(x)=g(x)=\left\{\begin{array}{l}
0, \\
1,
\end{array}\right.
$$$$
x \geqq d \text {, }
$$

with the further assumption that $g(x)$ is bounded for $0<x<d$.

Under the assumption that $f(x) \not \equiv g(x)$, set

$$
\Delta=\underset{x}{\operatorname{Max}}|f(x)-g(x)|,
$$

and let $y$ be the largest integer in $[0, d]$ for which the maximum, assumed nonzero, is attained.

If we let $p_{i}=p_{i}(y), q_{i}=q_{i}(y), p_{i}=p_{i}(y), \bar{q}_{i}=\bar{q}_{i}(y)$ be sets of values for which the min-max $=$ max-min is assumed, we have

$$
f(y)=T(p, q, f), \quad g(y)=T(\bar{p}, \bar{q}, g),
$$

and, as in Lemma 2 ,

$$
\Delta=|f(y)-g(y)| \leqq \operatorname{Max}_{p, g}[|T(p, q, f-g)|] .
$$

Since, for all $p$ and $q$,

$$
|T(p, q, f-g)| \leqq \Delta,
$$

we see that (16) is an equality, which means that

$$
T(p, q, f)=T(\bar{p}, q, f), \quad T(\bar{p}, \bar{q}, f)=T(\bar{p}, q, f) .
$$

Consider the relation

$$
\begin{aligned}
f(y)-g(y)= & \bar{p}_{1} q_{1}[f(y-1)-g(y-1)]+\bar{p}_{2} q_{1}[f(y+c)-g(y-c)] \\
& +\bar{p}_{1} q_{2}[f(y+a)-g(y+a)]+\bar{p}_{2} \bar{q}_{2}[f(y-b)-g(y-b)] .
\end{aligned}
$$

Since $\sum_{i, j} p_{i} q_{j}=1$ if any of the brackets in (19) have absolute value less 
than $\Delta$, the corresponding coefficient $p_{i} q_{j}$ must be zero. By assumption; $y$ was the largest value for which $|f(y)-g(y)|=\Delta$. Hence $p_{2} q_{1}=0, p_{1} q_{2}=0$.

Since $p_{1}+p_{2}=1$, both $p_{1}$ and $p_{2}$ cannot be zero, which means $q_{1}=0$ or $q_{2}=0$. Turning to the game matrix

$$
\left(\begin{array}{ll}
f(y-a) & f(y+a) \\
f(y+c) & f(y-b)
\end{array}\right),
$$

we see that the strict monotonicity of $f(x)$ as a function of $x$ makes it impossible for $q_{1}=0$ or $q_{2}=0$ to be optimal play at $x=y$. This yields a contradiction to $\Delta>0$ and completes the proof of uniqueness.

We see then that the proof of uniqueness of a strictly increasing solution is relatively easy, with the whole difficulty of the complete uniqueness proof centering about the proof of strict monotonicity.

The method we have employed is quite general and applies to large classes of functional equations. It fails, however, to treat the general case where we assume only that the elements $a_{i j}$ of the game matrix $A$ are real quantities.

\section{BIBLIOGRAPH Y}

1. K. J. Arrow, T. E Harris, and J. Marschak, Optimal inventory policy, Cowles Commission Paper No. 44, 1951.

2. R. Bellman, Bottleneck problems, functional equations and dynamic programming, Econometrica vol. 23 (1955) pp. 73-87.

3. - Dynamic programming and a new formalism in the theory of integral equations, Proc. Nat. Acad. Sci. U.S.A. vol. 41 (1955) pp. 31-34.

4. - Stability theory of differential equations, McGraw-Hill, 1953.

5. - A survey of the theory of boundedness, stability and asymptotic behavior of linear and nonlinear differential and difference equations, Office of Naval Research, 1949.

6. — Dynamic programming, mathematics for modern engineers, ed. by E. F. Beckenbach, McGraw-Hill, Chapter 12, to appear.

7. — Dynamic programming. A survey, Operations Research Quarterly vol. 2 (1954) pp. 275-284.

8. - The theory of dynamic programming, Nat. Proc. Acad. Sci. U.S.A. vol. 38 (1952) pp. 716-719.

9. - Some problems in the theory of dynamic programming, Econometrica vol. 22 (1954) pp. 37-48.

10. - Some functional equations in the theory of dynamic programming, Proc. Nat. Acad. Sci. U.S.A. vol. 39 (1953) pp. 1077-1082.

11. — The theory of dynamic programming, Bull. Amer. Math. Soc. vol. 60 (1954) pp. 503-515.

12. - On the optimal inventory equation, Management Science (to appear).

13. Dynamic programming and a new formalism in the calculus of variations, Proc. Nat. Acad. Sci. U.S.A. vol. 40 (1954) pp. 231-235.

14. - On computational problems in the theory of dynamic programming, Proc. Symposium on Numerical Methods, Santa Monica, 1953, New York, McGraw-Hill, 1955.

15. - Monotone convergence in dynamic programming and the calculus of variations, Proc. Nat. Acad. Sci. U.S.A. vol. 40 (1954) pp. 1073-1075.

16. R. Bellman and D. Blackwell, On a particular non-zero-sum game, September, 1949 (unpublished). 
17. R. Bellman and J. P. LaSalle, On non-zero-sum games and stochastic processes, August, 1949 (unpublished).

18. R. Bellman and S. Lehmann, On the continuous gold-mining equation, Proc. Nat. Acad. Sci. U.S.A. vol. 40 (1954) pp. 115-119.

19. - A functional equation in the theory of dynamic programming and its generalizations (submitted to Memoirs of the American Mathematical Society).

20. A. J. Dvoretzky, J. Kiefer, and J. Wolfowitz, The inventory problem-I: Case of known distributions of demand and The inventory problem-II: Case of unknown distributions of Demand, Econometrica vol. 20 (1952) pp. 187-222.

ThE RAND CORPORATION, Santa Monica, Calif. 